\title{
Application of CNC Engraving Process CAXA Software Platform based on Thin-walled Aluminum Parts
}

\author{
Zhu Xiurong \\ College of Mechanical Engineering, Jilin Teacher's Institute of Engineering and Technology, \\ Changchun, Jilin, china 130052 \\ Emai: zxr67811@163.com
}

\begin{abstract}
In this paper, CAD \ CAM technology applications in the engraving industry related research and analysis, CAXA software platform on aluminum alloy thin-walled parts made engraving applied research, Completed the process of design and processing, CNC machining tool selection and processing post-processing, Automatic generation of program, transfer to processing on CNC milling machine, and get a better CNC engraving effect o Carving processing enterprises provide an important reference.
\end{abstract}

Keywords- CAXA manufacturing engineer; Aluminum; Thin-walled parts; CNC technology; Engraving

\section{INTRODUCTION}

Industrial design is a creative activity, is the combination of science and art, which in the modern mode of production-based, relying on science and technology, the whole of creation reflects the visual representation of industrial products and a unified physical reproduction, along with industrial design development theory and practice, as well as science and technology widely used in the field of industrial design, industrial design make the techniques, methods has been greatly improved and updated, development and application of industrial design approach is hand-carved and machining combined with computer-aided manufacturing technology continues to mature, fully utilizing computer technology to complete relief engraving on CNC machine tool technology, not only can greatly improve production efficiency, and can significantly reduce costs and shorten the production cycle, to meet the needs of different industries, and has broad market prospects 。 [1]

CAD / CAM technology is based on computer and peripheral equipment and software-based technology, which comprises : Graphic graphic design , three-dimensional graphic modeling of design, analysis and optimization of the design of finite element, CNC programming, machining simulation and related parts and other products of data and management. Workers will be programmed computer applications in a whole new way of graphic design $0 \mathrm{CAD} / \mathrm{CAM}$ technology is fully converted before using only hand drawings and shop drawings as required to carry out the production and processing of system management approach, in which the most significant relief design applied to the image, the image embossed surface pattern shapes are often very complicated, there is no geometry, details more rules, the traditional manual processing is difficult to achieve, now the application of the results from the domestic point of view, mainly based on grayscale relief image 。[2]

\section{CAXA ENGRAVING SOFTWARE TECHNOLOGY}

CNC engraving technology is developed on the basis of computer-aided technology , computer-aided processing technology, computer numerical control technology, high-speed milling technology and laser technology and other related technologies over an engraving technique。Figure 1.1 shows several different stages of development experience carving technique, which inherits the lightweight fine engraving technology, comfortable and flexible processing advantages, while taking advantage of the traditional processing technology to automatically generate and put them in a perfect combination together, become a leader in the traditional carving techniques of carving a new processing technology, today's most advanced engraving configuration, but also led the most advanced engraving techniques。 


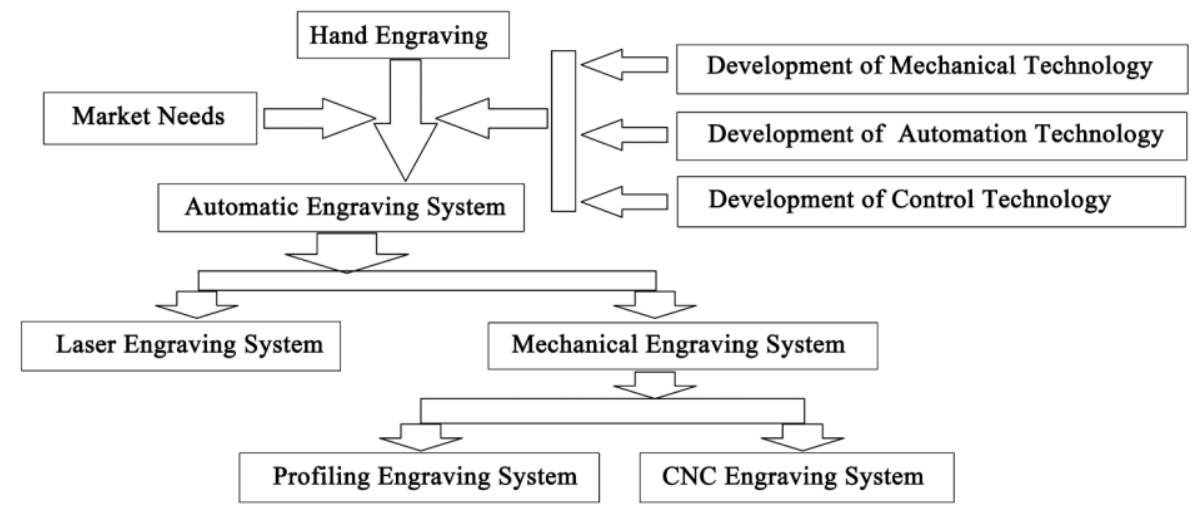

Figure 1. Phase engraving technology experience

III. CNC engraving technology based on CAXA software

Mainly for CAD / CAM software CAXA manufacturing engineer 2011 CNC engraving programming process conducted in-depth applied research 。 Completed the process of design, CNC machining tool selection and processing post-processing, Analysis of the operating personnel in CNC machining technology, CNC machining tool selection, $\mathrm{CNC}$ tool trajectory generation, processing and post-processing to ensure accuracy and so provide an important reference。

Picture selection and insertion

Select the relief image to be processed, the format BMP format as shown:

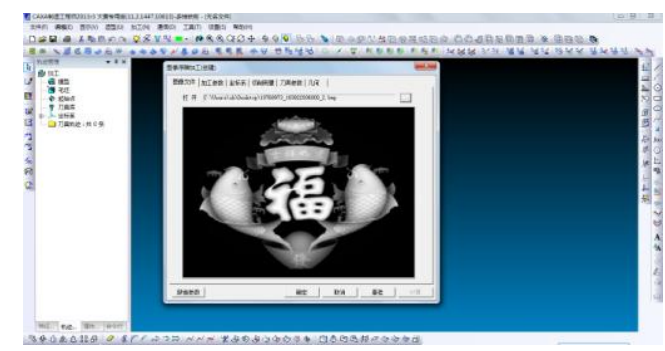

Figure 2. CAXA manufacturing engineer in the relief pattern inserted

\section{A. Choose material}

Choice of alloy material processing thin-walled parts, the CNC milling machining relief。Aluminum materials no crust, process better。Select the blank, establish the workpiece coordinate system as shown:

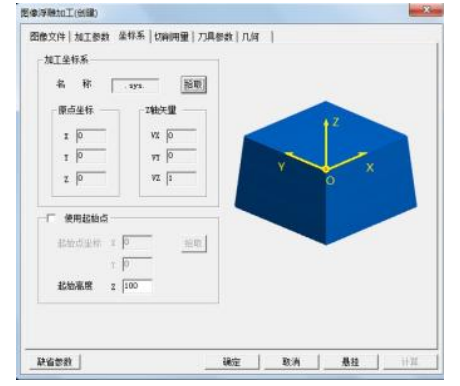

Figure 3. Establish the workpiece coordinate system

\section{B. Select the cutting parameters}

Image processing relief of cutting parameters and tool selection as shown:

Because parts of the blank choice is aluminum, so the processing performance of its cutting, the use of the processing machines used $\mathrm{CNC}$ milling machine, taking into account the actual use of $\mathrm{CNC}$ milling machines and machining performance characteristics, the knife back to eat the maximum amount of value is $0.6 \mathrm{~mm}$, eat the amount of cutting back the selected MID is $0.2 \mathrm{~mm}$, select finishing finishing allowance is $0.01 \mathrm{~mm}$ 。 Top height of $0 \mathrm{~mm}$, processing spacing $0.25 \mathrm{~mm}$, processing layers is 3 , the minimum torque step 0.2 , the height of the highest type of white, smooth frequency is 0 , the origin located in the center of the picture, the safe height $50 \mathrm{~mm}$, slow retraction height $15 \mathrm{~mm}$, take the knife to a reciprocating manner。[3] 


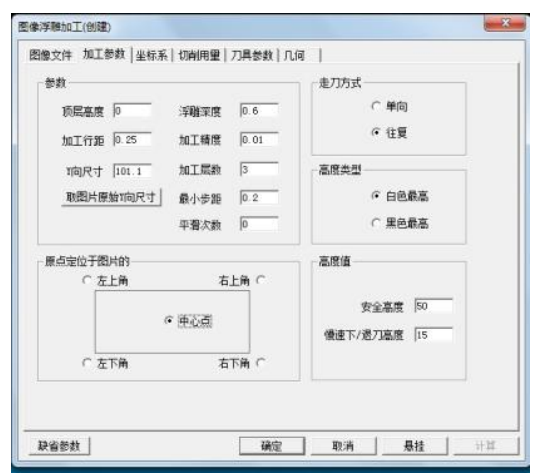

Figure 4. Select carving cutting parameters

\section{Cutting tool selection}

In actual production, machining of a part can not use a knife only, so you must create the tool according to the machining。

CAXA manufacturing engineer currently offers three cutters: ball cutter $(r=R)$, $R$ knife $(r<R)$, end knife $(r=$ $R$ ) where $R$ is the radius of the tool, $r$ is the radius of the knife angle, as well as tool parameters shank length $\mathrm{L}$ and the cutting edge length 1。 Tool parameters are as follows: [4]

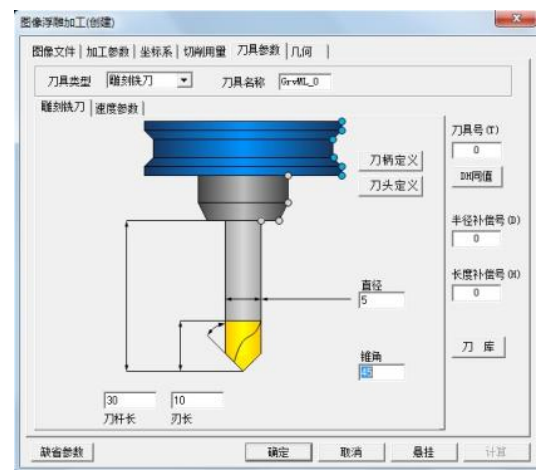

Figure 5. Tool parameters

\section{Image engraving trajectory generation}

CAXA manufacturing engineer grayscale image engraving is determined by the main image, in the process only through rational selection process of rational design and tools in order to generate a relatively perfect machining trajectory。Command generated by the image engraving tool path as shown: [5]

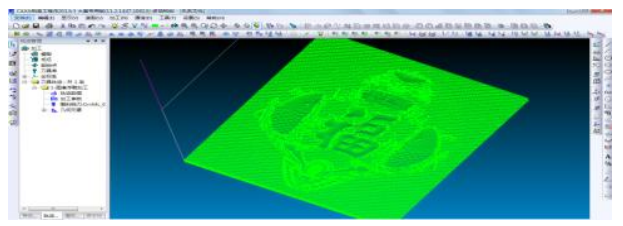

Figure 6. Image carving tracks

\section{E. Post Processing}

Rear set is for a particular machine, combined machine configuration already set on the rear output $\mathrm{NC}$ program format, such as the program line number, program size, data format, programming, arc control mode can be set 。 [6]

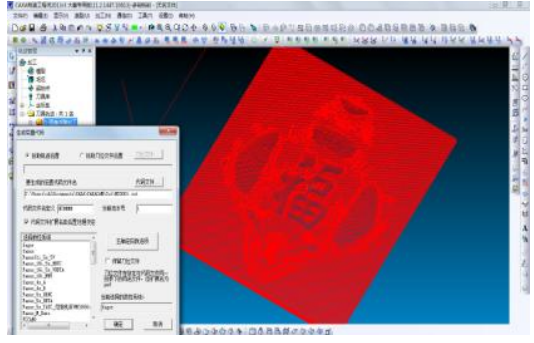

Figure 7. After the image configuration settings

F. CAXA manufacturing engineers simulate machining and program generation

$\mathrm{G}$ code is generated according to the current machine type configuration requirements, the already generated toolpath conversion generate G-code data file that the CNC program, with the NC program can be directly input NC machine tools 。 [7]

CAXA manufacturing engineer simulation capabilities intuitive and precise workpiece for machining process simulation and automatic code generation $\circ$ In the simulation process can be arbitrary scaling and rotation, to facilitate observation of the details of the process; simulation speed can also be adjusted; the results can process multiple paths simultaneously appearing; in the machining process simulation can be achieved machining tool shank interference, without cutting the interference situation retraction process (G00) of the machining tool interference and make the appropriate checks; you can cut the shape theory and simulation results are compared with each other and other parts 。 [8][9]

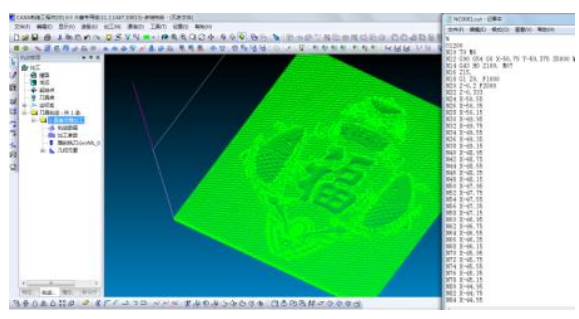

Figure 8. Simulation and code generation process

Through analysis of the main factors affecting the choice of cutting tools and machining tool selection principle should be considered, so that you can choose the correct sound processing tool。This helps: reduce the program's debugging time, work to reduce the time it takes to machine tools, shorten production cycle; can reduce the number and manual clamping tool changes brought about by human error and improve the accuracy of processing, improve the processing accuracy and the ability of machining complex workpieces ; after machining simulation and interference checking tools can be programmed so that a successful, greatly reducing the time and try to adjust the machine cutting time and increase the relative utilization of manufacturing resources。The resulting program transmission via data cable or IF card to CNC milling machines, cycle start, for online processing, and processing the ideal parts 。 [10] 


\section{Conclusion}

By CNC engraving examples, CAD / CAM software CAXA manufacturing engineer $2011 \mathrm{CNC}$ engraving programming process, conducted in-depth analysis. Completed the process of design, CNC machining tool selection and processing post-processing, and access to the ideal CNC machining results 。 This is the operator in $\mathrm{NC}$ machining process analysis, CNC machining tool selection, CNC tool trajectory generation, processing and post-processing to ensure accuracy and so provide an important reference 。

\section{REFERENCES}

[1]Han Liyang. Research on the Economic axis CNC engraving machine Key Technology[D]; Suzhou University; 2010.

[2]Zhang Dangfei. CNC engraving CAD CAXA based / CAM technology research[D]; Xi'an University of Architecture and Technology ; 2012
[3] Wang Yutao. Ceramic body CNC engraving processing technology [D]; Jingdezhen Ceramic Institute; 2014.

[4] Du Shiwei. Chinese characters carved NC code is automatically generated System [D]; Wuhan University ; 2011.

[5]Zhang Haiyan. Research and implementation of relief surface modeling [D]; Qingdao University; 2012.

[6]Liu Xin, Yu Rongbiao. Precision engraving technology in complex machining of mold surfaces $[\mathrm{J}]$; Precision Forming Engineering; 2011.03

[7]Bai Yongming. Application of CNC engraving process in purple plate interior Making [J]; New technologies; 2015.09

[8]Tang Zhengxiang. Relief triangular mesh surface editing and pasting Based[D]; Nanjing University of Aeronautics and Astronautics; 2011.

[9]Zhang Qunying, Zhang Ye, Guo Xiujie. Practical Teaching CNC engraving sharpening knife cone inquiry[J]; Gakuen; 2015.03

[10]Hu Xuyan, Zhang Wenjian. Discussion CNC engraving clamping method $[J]$; Journal of Tianjin Vocational Schools; 2012.10 